\title{
Magnetic property of a staggered-array undulator using a bulk high-temperature superconductor
}

\author{
Ryota Kinjo, Kenta Mishima, Yong-Woon Choi, Mohamed Omer, Kyohei Yoshida, \\ Hani Negm, Konstantin Torgasin, Marie Shibata, Kyohei Shimahashi, Hidekazu Imon, \\ Kensuke Okumura, Motoharu Inukai, Heishun Zen, Toshiteru Kii, Kai Masuda, \\ Kazunobu Nagasaki, and Hideaki Ohgaki \\ Institute of Advanced Energy, Kyoto University, Gokasho, Uji, Kyoto 611-0011, Japan
} (Received 11 September 2013; published 26 February 2014)

\begin{abstract}
The magnetic field of a staggered-array undulator using a bulk high-temperature superconductor is calculated by analytical and numerical methods. Analytical formulas for the undulator field and the solenoid field required to generate the undulator field are derived from a simple two-dimensional model. The analytical calculation shows the degree of dependence of these fields on the undulator parameters, the generation of a high undulator field proportional to the critical current density of the bulk superconductor, and the good tunability of the undulator field over a wide range of values. The numerical calculation is performed in a three-dimensional geometry by two methods: the center field and energy minimization methods. The latter treats the current distribution inside the bulk, whereas the former neglects it as a natural extension of the analytical model. The calculation also reveals the dependence of the fields on the undulator parameters arising from the current distribution. From the comparison with experimental results, we find that the latter method reproduces the experimental results well, which indicates the importance of the current distribution inside the bulk. Therefore, we derive a semiempirical formula for the required solenoid field by modifying the analytical formula using the numerical results so as to include the effect of the current distribution. The semiempirical formula reproduces the numerical result with an error of $3 \%$. Finally, we estimate the magnetic performance of the undulator as an example of using the formulas and values presented in this paper. The estimation shows that an undulator field twice as large as that of the present in-vacuum undulator but with an equal period and gap can be obtained at a temperature of approximately $20-40 \mathrm{~K}$, and that deflection parameters ( $K$ values) of 1 and 2 can be achieved with periods of 5 and $10 \mathrm{~mm}$ at approximately 4-20 K.
\end{abstract}

DOI: 10.1103/PhysRevSTAB.17.022401

PACS numbers: 07.85.Qe

\section{INTRODUCTION}

Synchrotron radiation from a relativistic electron beam passing through an undulator, i.e., undulator radiation, and free electron lasers (FELs) are crucial to a wide range of scientific applications. The fundamental wavelength of undulator radiation and FELs depends on the electron beam energy $E$, undulator period $\lambda_{u}$, and undulator field strength $B_{0}$. As undulators had typically had periods of $\mathrm{cm}$ order, the only way to obtain bright undulator radiation in the hard $\mathrm{x}$-ray region $(10-25 \mathrm{keV})$ had been to use highenergy electron beams in large synchrotron facilities such as the European Synchrotron Radiation Facility in France $(E=6 \mathrm{GeV})$, Advanced Photon Source in the U.S.

\footnotetext{
r-kinjo@spring8.or.jp

Present address: RIKEN SPring-8 Center, 1-1-1, Kouto, Sayo-Cho, Sayo-Gun, Hyogo 679-5148, Japan.

Published by the American Physical Society under the terms of the Creative Commons Attribution 3.0 License. Further distribution of this work must maintain attribution to the author $(s)$ and the published article's title, journal citation, and DOI.
}

(7 GeV), and SPring-8 in Japan $(8 \mathrm{GeV})$. A demonstration of $4.6 \mathrm{keV}$ x-ray generation from the $2.584 \mathrm{GeV}$ synchrotron in the National Synchrotron Light Source was performed with an 11-mm-period undulator [1]. Since then, moderate-energy synchrotron facilities with short-period undulators, such as the Swiss Light Source in Switzerland $\left(E=2.4 \mathrm{GeV}, \lambda_{u}=17 \mathrm{~mm}\right)$, DIAMOND in the U.K. ( $3 \mathrm{GeV}, 21 \mathrm{~mm})$, and SOLEIL in France $(2.75 \mathrm{GeV}$, $20 \mathrm{~mm}$ ), have been constructed [2-4]. In these facilities, the fundamental wavelengths of undulator radiation reach the $\mathrm{x}$-ray region; harmonics from the 5th through 11th reach the hard $\mathrm{x}$-ray region. This scheme also prevails for FELs. The first $\mathrm{x}$-ray laser of $0.12 \mathrm{~nm}(10 \mathrm{keV})$ was achieved at LCLS in the U.S. (15 GeV, $30 \mathrm{~mm}$ ) [5], and such lasers have already been used for x-ray science [6]. Subsequently, a $0.06 \mathrm{~nm}(20 \mathrm{keV})$ FEL was achieved at SACLA in Japan (8 GeV, $18 \mathrm{~mm}$ ) [7]. Recently, an x-ray FEL using the 3rd and 5th harmonics from a $3 \mathrm{GeV}$ electron beam source and a 15-mm-period undulator has been proposed [8]. As can be concluded from the above, short-period undulators require lower electron beam energies, meaning lower costs, a shorter construction time, and 
less effort, and also provide opportunities for scientists to use hard $\mathrm{x}$ rays in various applications. The undulator field strength, although not a determining parameter for the wavelength, must be sufficiently high to maintain the brilliance of the radiation, especially that of the harmonics, and the gain of amplification in FELs at a high level with a short-period undulator and low-energy electron beam. In addition, the use of multi-MeV gamma rays from highfield, short-period undulators to generate polarized positron beams via particle-antiparticle pair production in future linear colliders has been proposed [9].

To realize higher-field, shorter-period undulators, several different types of undulator have been studied. In-vacuum undulators (IVUs) [10], which have specially coated permanent magnet (PM) arrays inside a vacuum vessel, have been used in the aforementioned demonstration and in moderate-energy facilities. Cryogenic PM undulators (CPMUs) [11], in which the residual flux and coercivity of PMs are enhanced by cooling the magnets in an IVU to 100-150 K, have been developed and put in use. An undulator using a low-temperature superconductor (LTS), generally called a superconducting undulator before high-temperature superconductor (HTS) undulators were invented, has come under review. The insertion of LTS wires in a vacuum vessel has been proposed and demonstrated by Hezel et al. [12], who attempted to remove the thick wall of thermal insulation between the electron beam trajectory and the LTS wires.

Recently, bulk HTSs have been extensively studied; a trapped field of over $17 \mathrm{~T}$ was reported in a 26-mmdiameter, 15-mm-thick bulk HTS at 29 K [13]. An undulator using a bulk HTS has several advantages over other undulators: (1) a bulk HTS can produce fields over 10 times stronger than those of PMs even when the PMs are cooled; (2) the magnetic properties of bulk HTSs are continuing to improve, whereas those of PMs have reached a limit; (3) HTSs are more suitable than LTSs in highcurrent accelerators, in which the thermal input from the electron beam and the radiation itself is high; (4) assembly is easier than for an undulator using LTSs. However, to use bulk HTSs in undulators, one has to investigate methods of magnetizing bulk HTSs and generating a sinusoidal magnetic field, i.e., an undulator field. So far, various approaches to achieving this have been proposed. Cryoundulator plus (CU+) [14], in which bulk HTS rings are mounted on PMs to enhance the magnetic field in a CPMU, has been proposed and demonstrated. A superconducting PM undulator $[15,16]$, in which bulk HTSs are magnetized by a dipole field perpendicular to the beam axis, has also been developed.

We previously proposed and developed a bulk HTS staggered array undulator (BHSAU) [17], a schematic diagram of which is shown in Fig. 1. The principle of operation is as follows. When a solenoid field is applied to the bulk HTSs in the negative- $z$ direction, superconducting
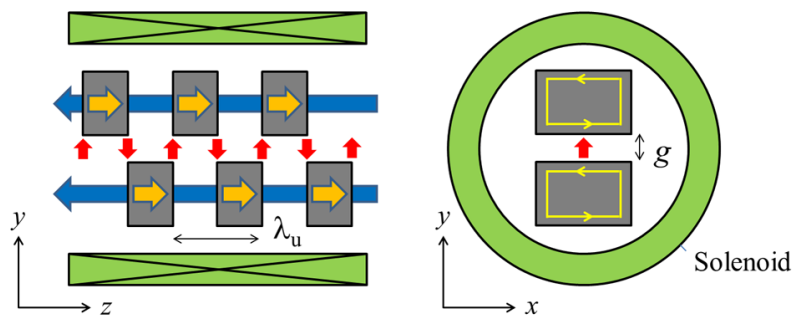

FIG. 1. Schematic view of BHSAU.

current loops are established to negate the change in the magnetic field inside the bulk HTSs; therefore, the bulk HTSs are magnetized in the positive- $z$ direction and thus generate a sinusoidal magnetic field in the $y$ direction. The BHSAU is based on a conventional staggered-array undulator (SAU) [18], in which soft magnetic blocks are in a staggered-array configuration instead of bulk HTSs. Therefore, the BHSAU inherits the properties of an SAU, i.e., tunability of the undulator field by the solenoid field. The advantages of the BHSAU are as follows. Because the undulator field is generated by bulk HTSs, which are magnetized in the same direction, one external solenoid can magnetize all the bulk HTSs and control the undulator field by controlling the magnetization of the bulk HTSs. Thus, a mechanical structure to control the gap is not required. Considering the large attraction force in a highfield undulator and the fact that cracking of the bulk HTSs occurred in the demonstration experiment of $\mathrm{CU}+$, this is a major advantage. So far, the demonstration of high-field generation at $6 \mathrm{~K}$ has been performed. An undulator field of $B_{0}=0.85 \mathrm{~T}$ was achieved at $6 \mathrm{~K}$ in a 6-periodic-number, 10-mm-period, 4-mm-gap prototype with a $2 \mathrm{~T}$ superconducting solenoid and a helium gas cooling system [19]. In the demonstration, we could only use a $2 \mathrm{~T}$ solenoid and rather small bulk HTSs because of budgetary restrictions. Even stronger undulators can be expected using a stronger solenoid.

Owing to a lack of formulas or computation codes to reproduce the undulator field of the BHSAU, the characteristics of the field inside the BHSAU have not been well studied. For a conventional PM undulator, the Halbach formula [20] can be used to easily estimate the undulator field. A three-dimensional magnetic field computation code, RADIA [21], which was developed in ESRF Insertion Device Laboratory, is commonly used to design undulators using PMs, ferromagnetic pieces, and/or electromagnetic coils. For an undulator using bulk HTSs, conventional methods cannot be used because of the difficulty of calculating the magnetization of a bulk HTS. The superconducting loop current inside a bulk HTS depends on the critical current density $J_{c}$, the current distribution, and the history of the applied field after the superconducting transition. To further complicate matters, $J_{c}$ at a certain point in a bulk HTS depends on the magnetic field at the point. For example, values of $J_{c}$ for a small 
TABLE I. Critical current density of QMG-GdBCO under magnetic field. Measurements were carried out by the dc magnetization method with a small sample $\left(\sim 0.2 \mathrm{~mm}^{3}\right)$.

\begin{tabular}{lcccc}
\hline \hline$T[\mathrm{~K}]$ & \multicolumn{4}{c}{$J_{c}\left[\mathrm{kA} / \mathrm{mm}^{2}\right]$} \\
$\left(T_{c}=94 \mathrm{~K}\right)$ & $B=0 \mathrm{~T}$ & $2 \mathrm{~T}$ & $4 \mathrm{~T}$ & $6 \mathrm{~T}$ \\
\hline 4 & 24 & 18 & 13 & 12 \\
10 & 16 & 11 & 8.7 & 8.2 \\
20 & 9.4 & 5.4 & 5.3 & 5.3 \\
40 & 3.5 & 2.2 & 2.4 & 2.2 \\
60 & 1.5 & 0.86 & 0.60 & $\cdots$ \\
\hline \hline
\end{tabular}

sample of QMG-GdBCO measured by the dc magnetization method are listed in Table I. Here, QMG-GdBCO from Nippon Steel Corporation [22] was the bulk HTS used in the experiment. To calculate the magnetization of bulk HTSs, there are multiple steps depending on the required accuracy. The first step is to use a simple current distribution that does not vary in the $z$ direction and a constant $J_{c}$ corresponding to the average critical current density in all bulk HTSs. The second is to use a current distribution that varies in the $z$ direction and a constant $J_{c}$. The third is to use a current distribution that varies in the $z$ direction and a $J_{c}$ that depends on the field.

In this paper, the magnetic properties of the BHSAU are studied. We develop an analytical model and derive analytical formulas for the undulator field and the solenoid field required to generate the undulator field. A constant $J_{c}$ and a simple current distribution are assumed. The basic characteristics of the fields are obtained using the formulas. Moreover, we develop a three-dimensional numerical model to precisely calculate these fields. The numerical computation is performed by two methods to investigate the effect of the current distribution. One method is to assume a simple current distribution, and the other method is to treat the $z$-direction distribution of the current. A comparison with experimental results is also made to evaluate the methods. To precisely estimate the fields without a large computation cost, a semiempirical formula for the solenoid field is derived from the analytical formulas using the result of comparing the analytical and numerical calculations. Finally, we estimate the magnetic performance of the undulator as an example of using the formulas.

\section{METHOD}

\section{A. Analytical calculation}

Here, we discuss the simple two-dimensional model used for the analytical calculation. If we assume that the bulk HTSs in the BHSAU are infinitely long in the $x$ direction and that the BHSAU has an infinite periodic number, the magnetic field can be calculated under a twodimensional approximation. Figure 2 shows the geometry used for the calculation. Here, $D_{y}$ and $D_{z}$ are the height and

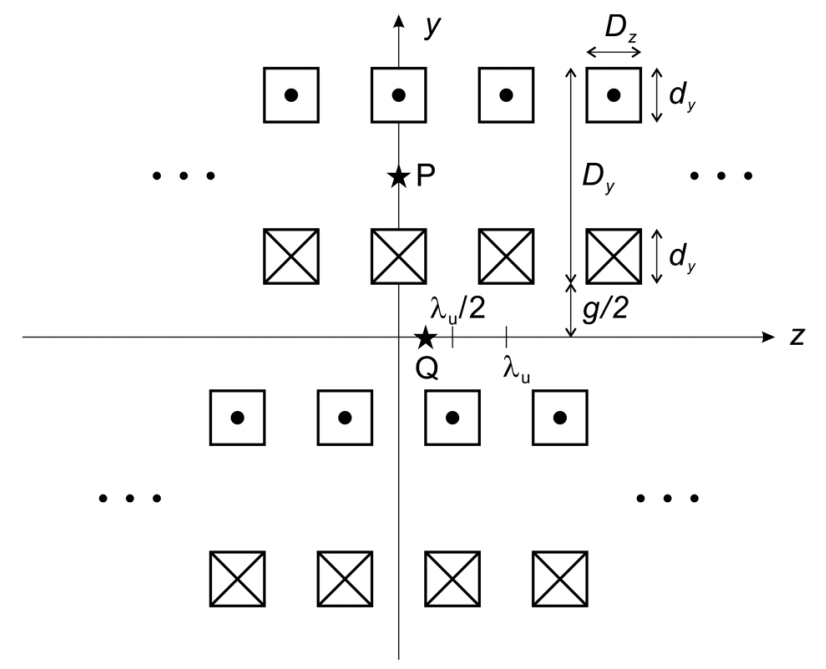

FIG. 2. Geometry used for analytical calculation.

thickness of the bulk, $d_{y}$ is the depth of the layer to which the current penetrates, and the coordinates of points $\mathrm{P}$ and $\mathrm{Q}$ are $(z, y)=\left(0,\left(D_{z}+g\right) / 2\right)$ and $\left(\lambda_{u} / 4,0\right)$, respectively. The centers of the bulk HTSs in the upper side are located at $z=n \lambda_{u}(n=\ldots,-1,0,1, \ldots)$ and those in the lower side are located at $z=(n+1 / 2) \lambda_{u}(n=\ldots,-1,0,1, \ldots)$. The amplitude of the undulator field is equal to the $y$ component of the field at point Q, i.e., $B_{0}=B_{y}(\mathrm{Q})$. On the basis of Bean's critical state model of a type-II superconductor [23], we assumed the following. The loop current flows inside the bulk from its outer edge; the layer containing the flowing current has depth $d_{y}$. The current density at all points is equal to the average constant critical current density, $J_{c}^{\text {bulk }}$, over the entire bulk. The loop current inside the superconductor flows to negate the change in the solenoid field; therefore, the $z$-direction field at point $\mathrm{P}$ is equal to the change in the solenoid field after the superconducting transition, i.e., $B_{z}(\mathrm{P})=-\Delta B_{s}$.

We next derive analytical formulas from the model. Using the two-dimensional Biot-Savart law, we have

$$
\begin{aligned}
& B_{0}= \mu_{0} J_{c}^{\text {bulk }} \lambda_{u} \sum_{n=1}^{\infty} \frac{\sin \left(n k_{u} D_{z} / 2\right)}{n^{2} \pi^{2}} \\
& \times e^{-n k_{u} g / 2}\left(1-e^{-n k_{u}\left(D_{y}-d_{y}\right)}\right)\left(1-e^{-n k_{u} d_{y}}\right) \\
&\left(0 \leq d_{y}<D_{y} / 2\right),
\end{aligned}
$$

$$
\Delta B_{s}=-\frac{\mu_{0} J_{c}^{\text {bulk }}}{2}\left[d_{y}+T\left(\lambda_{u}, g, D_{z}, D_{y}, d_{y}\right)\right], \quad T / d_{y}<10^{-3} .
$$

Here, $\mu_{0}$ is the vacuum permeability and $k_{u}$ is the wave number of the undulator field, i.e., $k_{u}=2 \pi / \lambda_{u}$. Writing $D_{z}=\lambda_{u} / 2$ and assuming $D_{y} \gg \lambda_{u}$, then, in practical units, 
$B_{0} \sim 0.13 J_{c}^{\mathrm{bulk}} \lambda_{u} \exp \left(-\pi \frac{g}{\lambda_{u}}\right)\left[1-\exp \left(-\frac{4 \pi \Delta B_{s}}{\mu_{0} J_{c}^{\mathrm{bulk}} \lambda_{u}}\right)\right]$.

Here, the units of $J_{c}^{\text {bulk }}$ and $\lambda_{u}$ are $\mathrm{kA} / \mathrm{mm}$ and $\mathrm{mm}$, respectively.

Next, we derive formulas to calculate the control curve of $B_{0}$. This is the $\Delta B_{s}-B_{0}$ curve used to control $B_{0}$ for wavelength tuning once $B_{0}$ reaches the target value of the operation. From Bean's critical state model, if we decrease $\Delta B_{s}$ from the target value, current in the reverse direction starts to flow from the outer edge of the bulk. Defining $d_{y}^{\downarrow}$ as the depth of the layer containing the current flowing in the reverse direction, then the magnetic field is described by the superposition of the magnetic fields generated by the positive current (whose depth is $d_{y}$ ) and the negative current (whose depth is $d_{y}^{\downarrow}$ ):

$B_{0}^{\downarrow}\left(d_{y}^{\downarrow}\right)= \begin{cases}B_{0}\left(J_{c}, d_{y}\right)-2 B_{0}\left(J_{c}, d_{y}^{\downarrow}\right) & \left(0<d_{y}^{\downarrow} \leq d_{y}\right) \\ -B_{0}\left(J_{c}, d_{y}^{\downarrow}\right) & \left(d_{y}<d_{y}^{\downarrow} \leq D_{y} / 2\right) .\end{cases}$

The formula for $\Delta B_{s}^{\downarrow}\left(d_{y}^{\downarrow}\right)$ is identical. If we stop decreasing $\Delta B_{s}$ and start to increase it again, current with the same direction as the original current starts to flow from the outer edge of the bulk. Defining $d_{y}^{\uparrow}$ as the depth of the layer in which this current flows, then the magnetic field is described by the superposition of the magnetic fields generated by the positive current (whose depths are $d_{y}$ and $d_{y}^{\uparrow}$ ) and the negative current (whose depth is $d_{y}^{\downarrow}$ ):

$$
B_{0}^{\uparrow}\left(d_{y}^{\uparrow}\right)= \begin{cases}B_{0}^{\downarrow}\left(d_{y}^{\downarrow}\right)+2 B_{0}\left(J_{c}, d_{y}^{\uparrow}\right) & \left(0 \leq d_{y}^{\uparrow} \leq d_{y}^{\downarrow}\right) \\ B_{0}\left(J_{c}, d_{y}\right) & \left(d_{y}^{\downarrow}<d_{y}^{\uparrow} \leq d_{y}\right) \\ B_{0}\left(J_{c}, d_{y}^{\uparrow}\right) & \left(d_{y}<d_{y}^{\uparrow} \leq D_{y} / 2\right) .\end{cases}
$$

The formula for $\Delta B_{s}^{\uparrow}\left(d_{y}^{\uparrow}\right)$ is identical.

\section{B. Numerical calculation}

\section{Three-dimensional model of bulk}

In this study, the path of the loop current inside the bulk is fixed and spatially discretized. Figure 3 shows the three-dimensional model of the rectangular bulk (upper) and D-shaped bulk (lower) considered in this study. The rectangular bulk is used for the comparison of the two methods and the comparison with the analytical calculation. Because a sufficiently large $D_{x}\left(D_{x}=10 \lambda_{u}\right)$

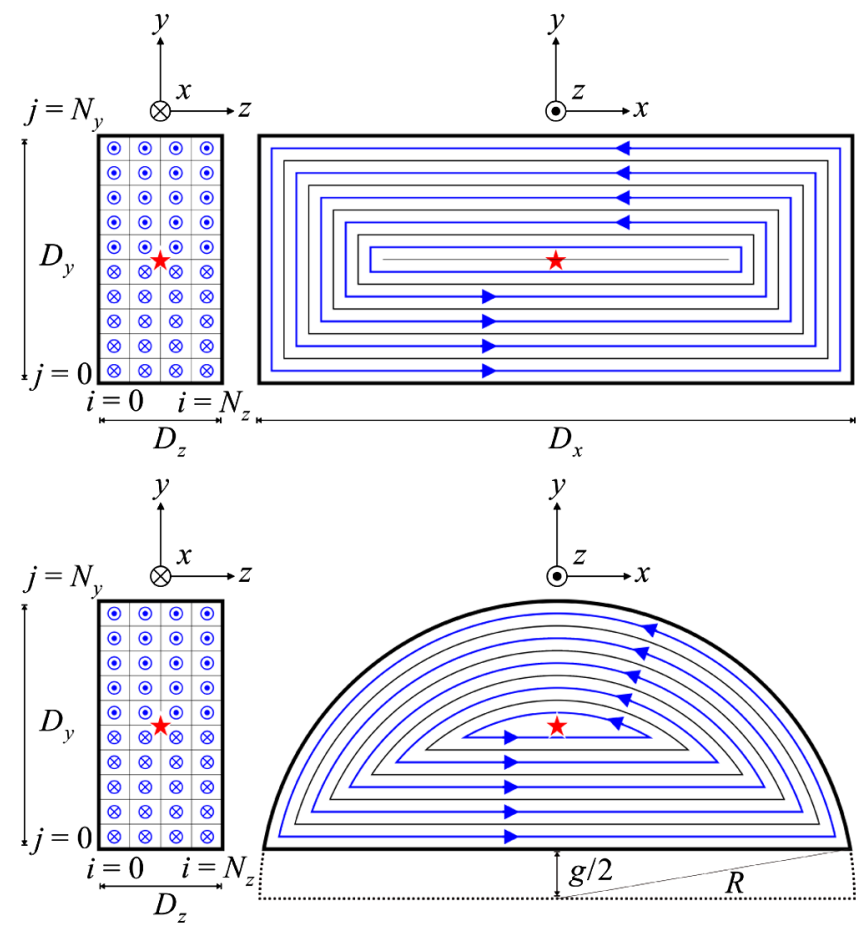

FIG. 3. Three-dimensional model of bulk HTS.

is assumed for the rectangular bulk, the calculation geometry is similar to that used in the two-dimensional analytical calculation $\left(D_{x} \rightarrow \infty\right)$. Also, $D_{y}=2 \lambda_{u}$ is commonly used in the comparison between the analytical and numerical calculations. Here, from Eq. (1), the maximum $B_{0}$ when $D_{y}=2 \lambda_{u}$ is over $99 \%$ of that when $D_{y} \rightarrow \infty$. The Dshaped bulk has the same shape as the bulk used in the experiment $(R=12.5 \mathrm{~mm})$ and is used for the comparison between the experiment and the numerical calculation. The left figures are cross-sectional views in the $y z$ plane and the right figures are cross-sectional views in the $x y$ plane. Here, $N_{z}$ and $N_{y}$ are the numbers of divisions of the bulks in the $z$ and $y$ directions, respectively. The stars indicate the center of the bulks. We assumed that the loop current flows only in the plane perpendicular to the $z$ axis. This is because the $z$ component is dominant in the solenoid field and in a field generated on a bulk HTS by other bulk HTSs. According to the assumption of Bean's critical state model for a type-II superconductor, the critical current density is constant throughout the bulk; thus, each loop has an equal current:

$$
I_{i j}=I_{c}=J_{c}^{\text {bulk }} \frac{D_{z} D_{y}}{2 N_{z} N_{y}}\left(0 \leq i<N_{y}, 0 \leq j<N_{z}\right) .
$$

\section{Center field method}

The basic idea of the center field (CF) method is the same as that used in the analytical calculation. The loop current flows to negate the change in the solenoid field at the center of the bulk. 
The key differences of this method from the analytical calculation are the finite periodic number and, for the Dshaped bulk, the three-dimensional geometry. Because of the finite periodic number, we assumed that each bulk HTS has a different depth at which the current flows, $d_{y, G}(G=1, \ldots, 2 N)$. Here, $N$ is the periodic number of the undulator and $2 N$ is the number of bulks. We also assumed that each depth is determined by the change in $B_{z}$ at the center of each bulk HTS, $B_{z}\left(\mathbf{r}_{c, G}\right)$. By defining $\mathbf{B}_{G H}$ as the magnetic field generated by the $H$ th bulk at the center of the $G$ th bulk, we obtained $B_{z}\left(\mathbf{r}_{c, G}\right)$ as

$$
B_{z}\left(\mathbf{r}_{c, G}\right)=\left[\sum_{H=1}^{2 N} \mathbf{B}_{G H}\left(d_{y, H}\right)\right]_{z}
$$

By using the above-mentioned assumption $B_{z}\left(\mathbf{r}_{c, G}\right)=$ $-\Delta B_{s}$,

$$
\left[\sum_{H=1}^{2 N} \mathbf{B}_{G H}\left(d_{y, H}\right)\right]_{z}+\Delta B_{s}=0 .
$$

To determine all $d_{y, G}$, an iterative computation was performed.

In the computation, $d_{y}$ is discretized by $n_{y}$. Here, $n_{y}$ is the discretized depth of the layer in which the current flows $\left(0 \leq n_{y} \leq N_{y}\right)$. Moreover, to obtain a high-precision result with a small number of divisions in the $y$ direction $\left(N_{y}\right)$, we assumed that the current $I_{c}$ flowed in $n_{y}$ lines and that a current of $\alpha I_{c}(0 \leq \alpha<1)$ flowed in the $\left(n_{y}+1\right)$ th line. Here, $\alpha$ is calculated from the residual $\Delta B_{s}-B_{z}\left(\mathbf{r}_{c, G}\right)$ in each iteration. Then, the field at the center of the $G$ th bulk generated by loop $i j$ in the $H$ th bulk is obtained using the discretized Biot-Savart law:

$$
\mathbf{B}_{G H i j}=\frac{\mu_{0} I_{c}}{4 \pi} \sum_{p=1}^{N_{c}} \frac{\Delta \mathbf{s}_{p} \times \mathbf{r}}{r^{3}} .
$$

Here, $N_{c}$ is number of the line elements (the number of divisions of the loop), $\Delta \mathbf{s}_{p}$ is the $p$ th line element of loop $i j$ in the $H$ th bulk, and $r$ is the distance between $\mathbf{r}_{c, G}$ and the center of the line element. Then, the field $\mathbf{B}_{G H}$ is expressed by

$$
\mathbf{B}_{G H}\left(n_{y, H}\right)=\sum_{i=1}^{N_{z}}\left(\sum_{j=1}^{n_{y, H}} B_{G H i j}+\alpha B_{G H i\left(n_{y, H}+1\right)}\right) .
$$

\section{Energy minimization method}

The energy minimization (EM) method was proposed by Badía et al. to determine the critical state in which a system organizes itself [24]. Sanchez and Navau applied the method to a single bulk HTS with a finite height and cylindrical symmetry [25]. The shielding current distribution of a finite cylinder can be obtained with a constant $J_{c}$ or field-dependent $J_{c}$ under a uniform/ nonuniform applied field.

In this study, to use the EM method for the threedimensional problem of bulk HTSs with an arbitrary shape, we assumed the path of the loop current and used the discretized Neumann formula to compute the inductance.

The EM method is as follows. The energy required for the current to flow is the sum of the work required for the current to flow against the magnetic field induced by other loops and the energy obtained upon the vanishing of the external field. For current $I_{i j}$ to flow in loop $i j$, the required energy is written as

$$
\begin{aligned}
E_{i j} & =I_{i j}\left(\Phi_{i j}^{\mathrm{int}}-\Phi_{i j}^{\mathrm{ext}}\right) \\
& =I_{i j}\left(\sum_{k l}^{N_{y} N_{z}} M_{i j, k l} I_{k l}-\int_{S_{i j}} B_{\text {ext }} \mathrm{d} S\right) .
\end{aligned}
$$

Here $\Phi_{i j}^{\mathrm{int}}$ is the magnetic flux on the surface surrounded by loop $i j$ generated by the other loops, $\Phi_{i j}^{\text {ext }}$ is the magnetic flux by the external field on the same surface, and $M_{i j, k l}$ is the mutual inductance $(i j \neq k l)$ or self-inductance $(i j=k l)$ between the loops. If a constant $J_{c}$ is assumed, $I_{i j}$ and $I_{k l}$ are both equal to $I_{c}$. The calculation procedure is as follows: (1) Calculate $E_{i j}$ for every loop that does not have a current. (2) Finish the calculation if there is no loop with $E_{i j}<0$. (3) Find the loop with the minimum $E_{i j}$. (4) Flow a current in the loop. (5) Repeat the calculation from step (1). The current distribution after the calculation shows the critical state.

The model can be applied to three-dimensional problems with bulk HTSs of an arbitrary shape if the path of the loop current is determined in advance. We assumed the current path shown in Fig. 3 in common with that in the $\mathrm{CF}$ method. The current flows in a fixed plane normal to the $z$ axis. This is similar to the thin-film approximation used in finite element analysis for bulk HTSs. However, in the thinfilm approximation, the current path is free in the plane normal to the $z$ axis; in this model, the current path is fixed in the plane to calculate the inductances in advance.

For a loop current with an arbitrary shape, it is difficult to find analytical formulas for the mutual inductance and self-inductance. Therefore, we numerically calculated the mutual inductance and self-inductance. The vector potential generated by loop $k l$ at position $\mathbf{r}$ is described by the Biot-Savart law:

$$
\mathbf{A}_{k l}(\mathbf{r})=\frac{\mu_{0} I_{k l}}{4 \pi} \oint_{C_{k l}} \frac{\mathrm{d} \mathbf{s}_{2}}{r} .
$$

Here $I_{k l}$ is the current flowing in loop $k l$ and $C_{k l}$ is the integration path on the loop. The magnetic flux generated by loop $k l$ on the surface surrounded by loop $i j$ is expressed by 


$$
\begin{aligned}
\Phi_{i j, k l} & =\oint_{C_{i j}} \mathbf{A}_{k l} \cdot \mathrm{d} \mathbf{s}_{1} \\
& =\frac{\mu_{0} I_{k l}}{4 \pi} \oint_{C_{i j}} \oint_{C_{k l}} \frac{\mathrm{d} \mathbf{s}_{1} \cdot \mathrm{d} \mathbf{s}_{2}}{r} .
\end{aligned}
$$

By discretizing the formula, we obtain the mutual inductance between loops $i j$ and $k l$ :

$$
M_{i j, k l}=\frac{\Phi_{i j, k l}}{I_{k l}}=\frac{\mu_{0}}{4 \pi} \sum_{p}^{N_{c}} \sum_{q}^{N_{c}} \frac{\Delta s_{i j p} \Delta s_{k l q}}{r_{p q}} .
$$

Here $\Delta s_{i j p}$ and $\Delta s_{k l q}$ are respectively the $p$ th and $q$ th line elements of loops $i j$ and $k l, r_{p q}$ is the distance between the centers of the two elements, and $N_{c}$ is the number of line elements (the number of divisions of the loop). The selfinductance of loop $i j$ is calculated as the mutual inductance between loop $i j$ and a slightly larger copy of loop $i j$, which is at a distance of $0.78 D_{y} / 2 / N_{y}$ from loop $i j$. For a torus with major radius $R_{i j}$ and minor radius $D_{y} / 2 / N_{y} / 2$, the calculation using this distance matches the analytical solution of the self-inductance. For a bulk HTS with an arbitrary shape, we define $R_{i j}$ as the smallest distance in the $y$ direction between loop $i j$ and the center of the bulk HTS (the star in Fig. 3).

\section{CALCULATION RESULTS AND COMPARISON WITH EXPERIMENT}

\section{A. Analytical results}

Equation (3) shows that the BHSAU has a much higher undulator field than conventional undulators at a high critical current density. As examples, the maximum undulator fields obtained with three representative parameter sets are shown in Table II. One can obtain the maximum $B_{0}$, i.e., $B_{0, \max }$, by substituting the value of $J_{c}$ listed in Table I. Here, parameter set (a) comprises values for the IVU in SACLA, (b) comprises those in our experimental setup, and (c) comprises those giving our target period.

Figure 4 shows the dependence of $B_{0}$ on $\Delta B_{s}$ at $\lambda_{u}=$ $10 \mathrm{~mm}$ and $g=4 \mathrm{~mm}$. The upper figure shows the initial curves for various $J_{c}^{\text {bulk }}$. The plot was obtained from Eqs. (1) and (2) by using $d_{y}$ as a parameter. The lower figure shows (1) the initial curve and sample control curves, i.e., (2) a curve to decrease $B_{0}$ and (3) a curve to increase $B_{0}$ again. The plot was obtained from Eqs. (4) and (5) using $d_{y}$,

TABLE II. Examples of maximum $B_{0}$ and $\Delta B_{s}$ (analytical).

\begin{tabular}{lcccc}
\hline \hline & & & $B_{0, \max } / J_{c}$ & $\Delta B_{s, \max } / J_{c}$ \\
& $\lambda_{u}[\mathrm{~mm}]$ & $g[\mathrm{~mm}]$ & {$\left[\mathrm{T} / \mathrm{kA} / \mathrm{mm}^{2}\right]$} & {$\left[\mathrm{T} / \mathrm{kA} / \mathrm{mm}^{2}\right]$} \\
\hline (a) & 18 & 3.6 & 1.26 & 13.4 \\
(b) & 10 & 4 & 0.364 & 7.45 \\
(c) & 5 & 1 & 0.350 & 3.72 \\
\hline \hline
\end{tabular}
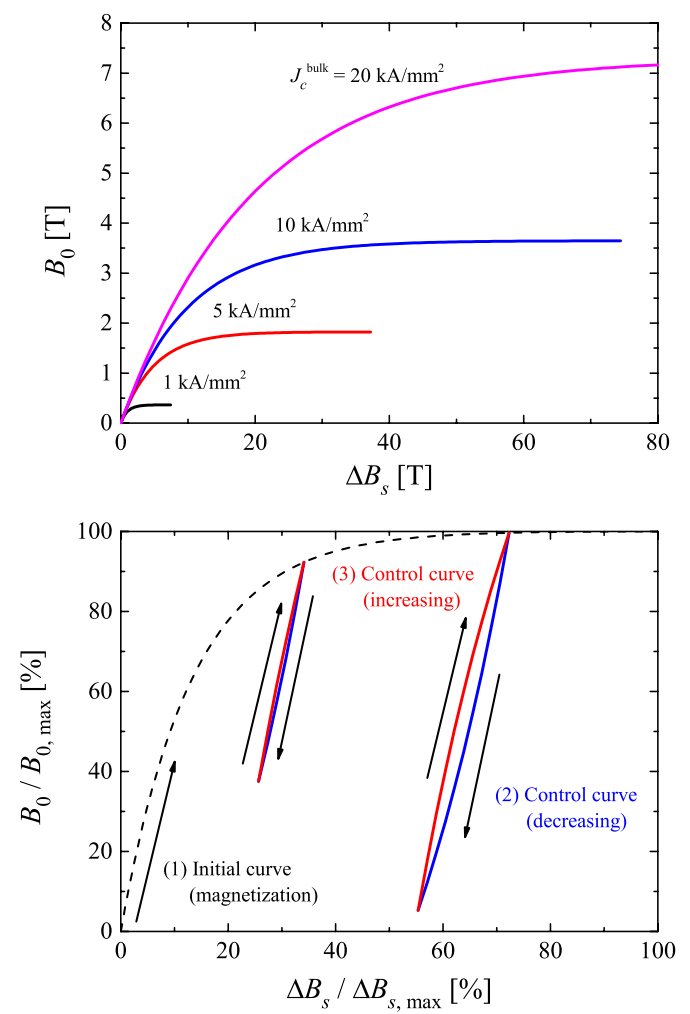

FIG. 4. Dependence of $B_{0}$ on $\Delta B_{s}$ (analytical, $\lambda_{u}=10 \mathrm{~mm}$, $g=4 \mathrm{~mm}$ ). The upper figure shows the initial curves for various $J_{c}^{\text {bulk }}$. The lower figure shows the initial curve and the sample control curves.

$d_{y}^{\downarrow}$, and $d_{y}^{\uparrow}$ as parameters. Here, the initial curve is the curve when the bulk is magnetized after the superconducting transition, and the control curves are the curves used to tune the wavelength of the undulator radiation during operation. There was hysteresis in the $\Delta B_{s}-B_{0}$ curve.

Table III shows values of $B_{0}$ and the corresponding values of $\Delta B_{s}$ required to obtain each $B_{0}$. Here, $\Gamma_{y}$ is defined as $\Gamma_{y}=2 d_{y} / D_{y}$ and is the depth of the fieldpenetrated layer in which the current flows. The values in this table have an error within $2 \%$ over a wide range of conditions $\left(0.1 \leq g / \lambda_{u} \leq 10\right)$.

TABLE III. Examples of values of $B_{0}$ and the corresponding values of $\Delta B_{s}$ required to obtain each $B_{0}$ (analytical, $B_{0} / B_{0, \max }$ has an error of $2 \%$ in the range $0.1 \leq g / \lambda_{u} \leq 10$ ).

\begin{tabular}{lcc}
\hline \hline$\Gamma_{y}[\%]$ & $B_{0} / B_{0, \max }[\%]$ & $\Delta B_{s} / \Delta B_{s, \max }[\%]$ \\
\hline 5 & 29 & 4.2 \\
10 & 48 & 8.5 \\
15 & 63 & 12.7 \\
20 & 72 & 17.0 \\
25 & 80 & 21.2 \\
30 & 86 & 25.5 \\
100 & 100 & 100 \\
\hline \hline
\end{tabular}




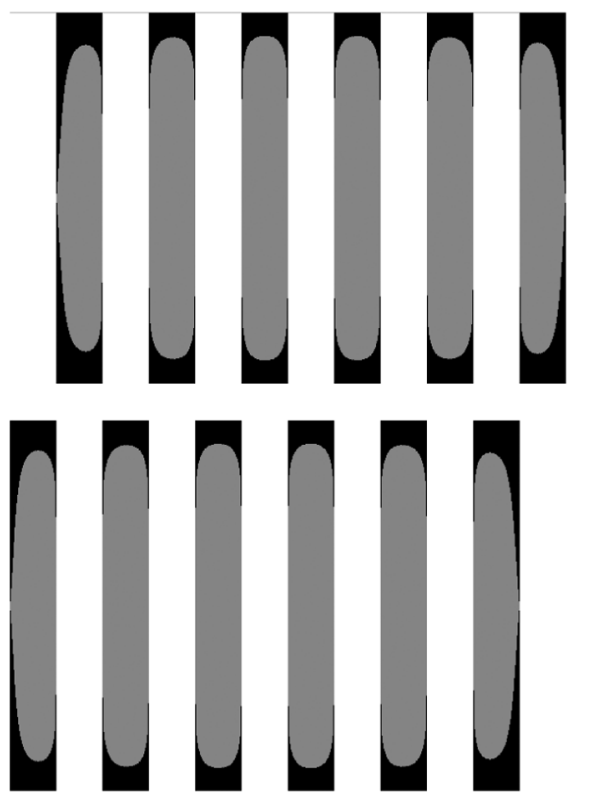

FIG. 5. Cross-sectional view of current distribution (EM method). The black area indicates where the current is flowing. The gray area indicates where the current is not flowing.

\section{B. Numerical results}

Figure 5 shows a cross-sectional view of the current distribution ( $x$-direction current in $z y$ plane) in the BHSAU with $N=6$ obtained by the EM method. The black area indicates where the current is flowing and the gray area indicates where the current is not flowing. The depths of the layer in which the current flowed at the bulk edge and bulk center were markedly different, even in the bulk near the undulator center. The bulk HTSs at the end of the undulator showed an asymmetric distribution of the depths. The depth of the layer in which the current flowed in the single bulk was flat in the calculation by the $\mathrm{CF}$ method, as was expected from the assumption. However, without relation to the difference in the current distributions, the total amounts of the current in the bulk near the center had at most $1 \%$ difference between the CF and EM methods.

Figure 6 shows the dependence of $B_{0}$ on the parameter $f$, which is defined by $f=1-D_{z} / \lambda_{u}$ : the ratio of the horizontal gap between two bulk HTSs to the period. The numerical calculation was performed by the CF and EM methods with $N=12$. To compare the numerical and analytical results, the numerical (analytical) fields at various $f$ were normalized by the numerical (analytical) value for $f=0.5$. In the analytical calculation and the numerical calculation by the CF method, for both $f<0.5$ and $f>0.5$ almost the same degradation of $B_{0}$ was observed for all $\Delta B_{s}$. In contrast, in the numerical calculation by the EM method, the $f>0.5$ condition sometimes resulted in a higher $B_{0}$ than the $f=0.5$ condition at low $\Delta B_{s}$.

Figure 7 shows the difference in the analytical and numerical values of $\Delta B_{s}$ required for the same $B_{0}$. Here, the
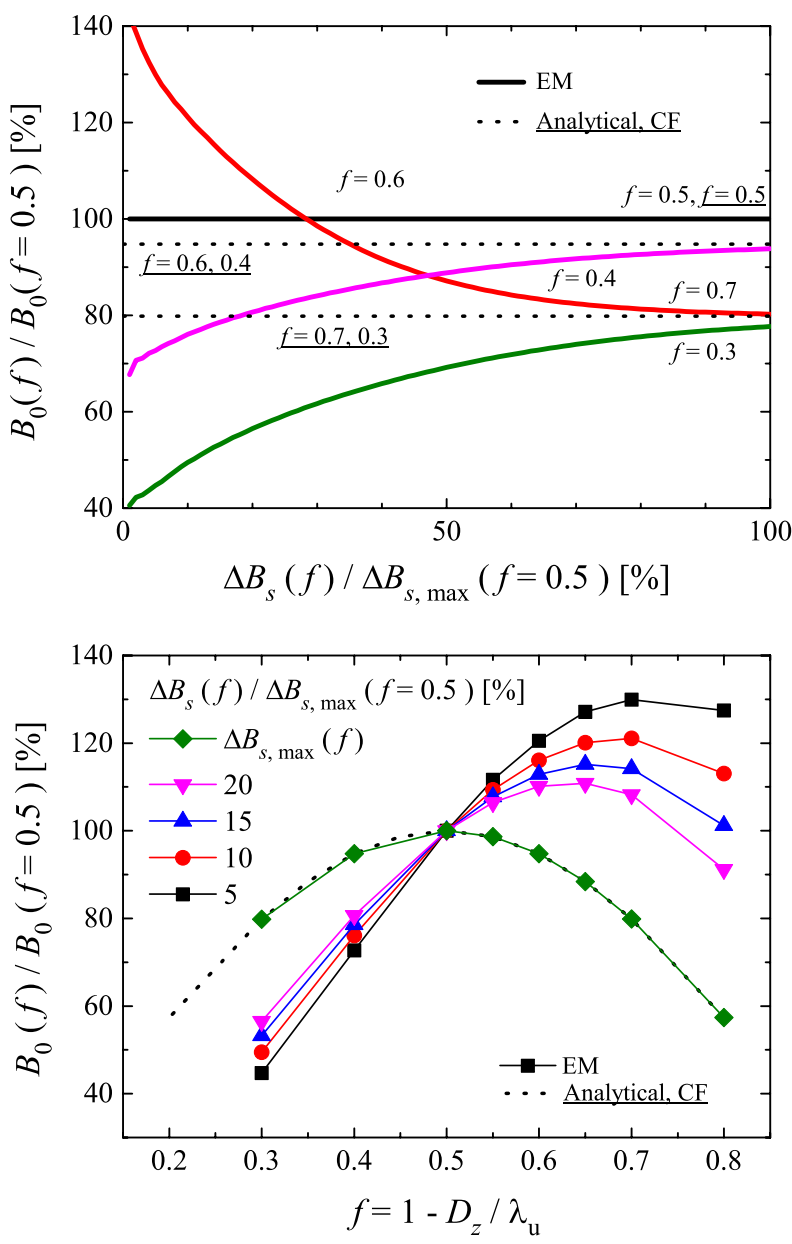

FIG. 6. Dependence of $B_{0}$ on $f$ (analytical, CF, and EM, $g / \lambda_{u}=0.4$ ). For comparison, the analytical (numerical) fields at various $f$ are normalized by the analytical (numerical) value for $f=0.5$.

superscripts An and $\mathrm{Nu}$ indicate analytical and numerical values, respectively. The numerical calculation was performed by the EM method with $N=12 . B_{0}^{\mathrm{An}}$ and $\Delta B_{s}^{\mathrm{An}}$ are the values respectively obtained by Eqs. (1) and (2). In the numerical calculation, a larger $\Delta B_{s}$ than the analytical value was required to generate the same $B_{0}$, particularly for low $B_{0}$. For $f=0.5$, the numerical value of $\Delta B_{s}$ required was up to $50 \%$ higher than the analytical value. For $f>0.5$, the requirement was moderate at low $B_{0}$. The divergence at high $B_{0}$ for $f>0.5$ means that $B_{0}(f)$ $(f>0.5)$ cannot reach $B_{0, \max }(f=0.5)$.

\section{Comparison between experiment and calculation}

The experiment was performed with a 6-periodicnumber, 10-mm-period, 4-mm-gap prototype with a $2 \mathrm{~T}$ superconducting solenoid and a helium gas cooling system. To equalize the geometry, the calculation was performed with a D-shaped bulk with the same size as that used in the experiment, $f=0.5, N=6, \lambda_{u}=10 \mathrm{~mm}$, and $g=4 \mathrm{~mm}$. 


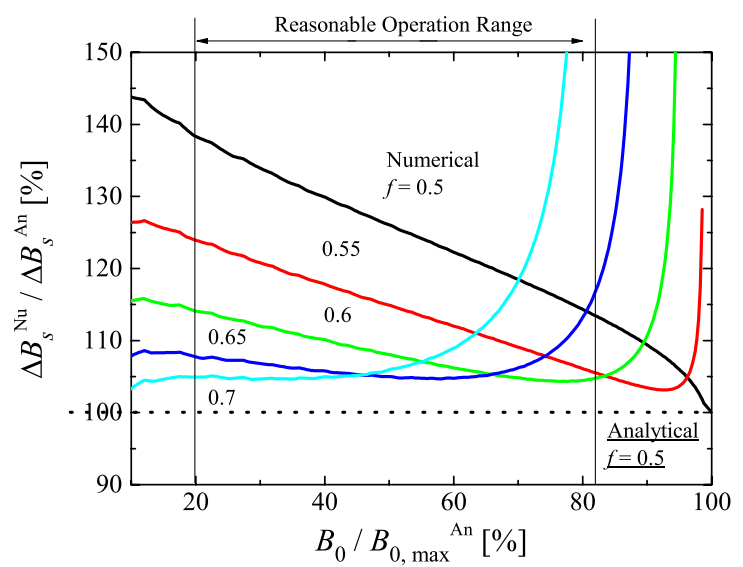

FIG. 7. Difference in the analytical and numerical values of $\Delta B_{s}$ required for the same $B_{0}$ (analytical and EM, $g / \lambda_{u}=0.4$ ). The superscripts $\mathrm{An}$ and $\mathrm{Nu}$ indicate analytical and numerical values, respectively. The numerical calculation was performed by the EM method with $N=12$.

The experimental results have an error of $4 \%$ owing to the measurement error. The setup of the experiment is described in Ref. [19].

Figure 8 shows a comparison of the experimental and calculated solenoid field dependences of the undulator field. The experimental results show data obtained at $T=20,40$, and $60 \mathrm{~K}$. The calculation results show data obtained at $J_{c}=10,5,2$, and $1 \mathrm{kA} / \mathrm{mm}^{2}$. The green dotted lines indicate the results obtained by the CF method and the pink solid lines indicate the results obtained by the EM method. The initial gradients of the curves, $\mathrm{d} B_{0} /\left.\mathrm{d} \Delta B_{s}\right|_{\Delta B_{s}=0}$ obtained by the experiment were 0.24 for all $T$, and those obtained by the EM and CF methods were 0.24 and 0.4 , respectively, for all $J_{c}^{\text {bulk }}$.

Figure 9 shows a comparison of the experimental and calculated field distributions of $B_{y}$ along the $z$ axis. In the experiment, after the prototype was cooled to $20 \mathrm{~K}$ under a solenoid field of $B_{s}=-2 \mathrm{~T}$, the solenoid field was

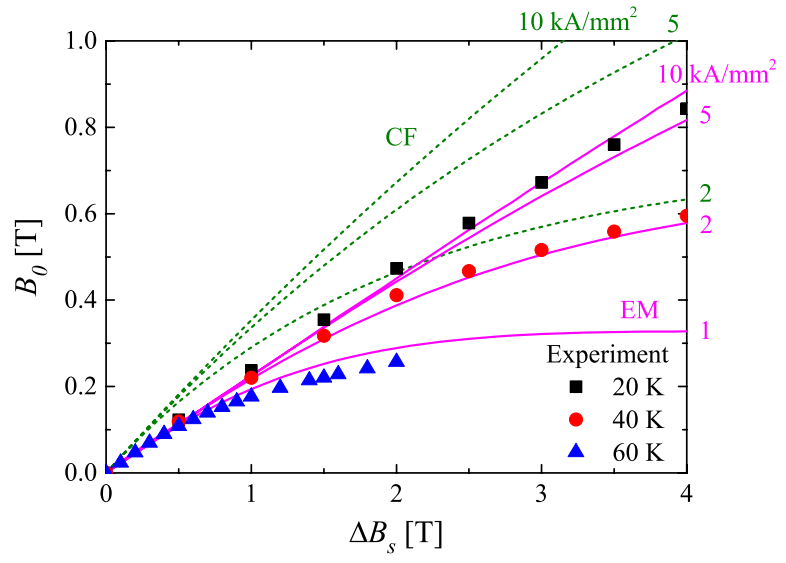

FIG. 8. Comparison of experiment and calculation: dependence of $B_{0}$ on $\Delta B_{s}$.

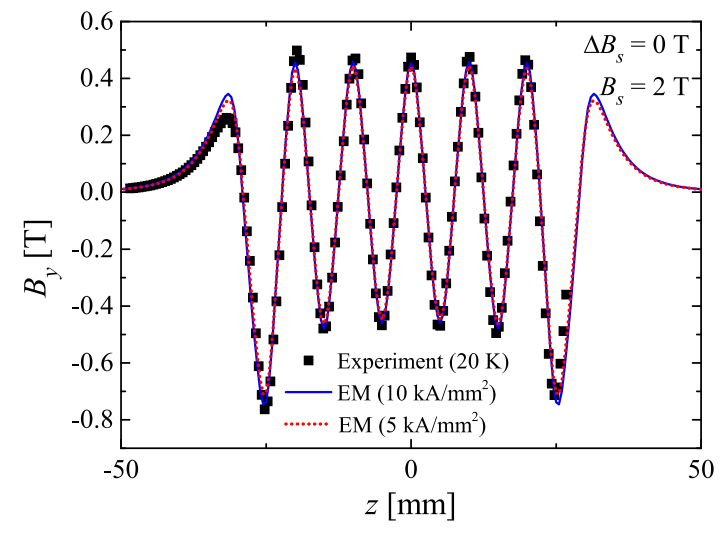

FIG. 9. Comparison of experiment and calculation: $B_{y}$ distribution along the $z$ axis.

changed to $B_{s}=0 \mathrm{~T}\left(\Delta B_{s}=+2 \mathrm{~T}\right)$ at $T=20 \mathrm{~K}$. This is known as field cooling. The calculation was performed by the EM method with $J_{c}=5$ and $10 \mathrm{kA} / \mathrm{mm}^{2}$.

\section{DISCUSSION}

\section{A. Basic characteristics of fields}

Here we clarify the characteristics of the BHSAU from the analytical calculations. Equation (1) shows that the undulator field of the BHSAU has common properties with that of a conventional Halbach-type PM undulator [20], such as the exponential dependence of the field on the gap and on the height of the magnet. Meanwhile, the undulator field of the BHSAU has the unique property that it depends on $J_{c}^{\text {bulk }}, \lambda_{u}$, and $d_{y}$. Equation (3) also shows that the BHSAU has a much higher undulator field than conventional undulators at a high $J_{c}^{\text {bulk }}$. Although its performance will be estimated later, here we introduce one example. If $J_{c}$ is over $2 \mathrm{kA} / \mathrm{mm}^{2}, B_{0, \max }$ under condition (a) is twice as large as the peak field in the present IVU $\left(B_{0}=1.30 \mathrm{~T}\right.$ at $\lambda_{u}=18 \mathrm{~mm}, g=3.6 \mathrm{~mm}$ ). From Fig. 4 , we obtain two findings. One is that the controllability of $B_{0}$ by the solenoid is maintained even in the saturation region in which $B_{0}$ is close to $B_{0, \max }$. This is a feature different from a conventional SAU, which has little controllability in the saturation region, and is beneficial for tuning the wavelength. The other is that the $\Delta B_{s}$ required to obtain $B_{0, \max }$ at a high $J_{c}$ is greater than that for present commercially available solenoids. Fortunately, as shown in Table III, there is a "reasonable operation range" in which $\Delta B_{s} / \Delta B_{s, \max }$ is much less than $B_{0} / B_{0, \max }$. For example, almost half the value of $B_{0, \max }, B_{0} / B_{0, \max }=$ $48 \%$ can be obtained even when $\Delta B_{s} / \Delta B_{s, \max }=8.5 \%$. Thus, for high $J_{c}$, the undulator should be operated in the reasonable operation range.

\section{B. Dependence on $f$}

We next discuss the additional properties of the BHSAU obtained from the numerical calculation by the EM method 
that were not observed in the analytical calculation and the numerical calculation by the $\mathrm{CF}$ method. One of the differences is the dependence of $B_{0}$ on $f$. As shown in Fig. 6, a higher $B_{0}$ was observed for $f>0.5$ than when $f=0.5$ for the same value of $\Delta B_{s}$. Because the CF method and the analytical calculation did not exhibit this phenomenon, it is not due to the effect of the finite periodic number but to the current distribution inside the bulk HTSs shown in Fig. 5. Here we discuss this in detail. Currents with two different directions that are symmetric with respect to the $z$ axis generate equal values of $B_{y}$ on the $z$ axis with opposite signs. When $f<0.5$, the number of such pairs increases with increasing bulk thickness; therefore, $B_{0}$ decreases. However, currents with two different directions that are symmetric with respect to a certain point on the $z$ axis generate the same $B_{y}$ at the point. When $f>0.5$, the numbers of such pairs decrease with decreasing bulk thickness; therefore, $B_{0}$ also decreases when $f>0.5$, similarly to when $f<0.5$. However, only in the EM method is there an effect that increases $B_{0}$ when $f>0.5$. In the EM method, the depth of the layer in which the current flows is large at the edge of the bulk. If the position of the edge moves in the $z$ direction to a point far from the field peak, the absolute field at the point decreases, although the $B_{y}$ component increases in some cases. Therefore, in the EM method, $B_{0}$ increases or decreases depending on the current distribution at a certain $\Delta B_{s}$ when $f>0.5$. This dependence on $f$ is opposite that for a conventional SAU, in which the undulator field is high at $f<0.5$ [26].

\section{Effect of current distribution}

Here we evaluate the agreement between the calculation and experiment. It is difficult to compare the $B_{y}$ profile or $B_{0}$ at a single value of $\Delta B_{s}$ without the exact value of the average $J_{c}$ in the experiment. Thus, we used the dependence curve of $B_{0}$ on $\Delta B_{s}$. As is clear from Eqs. (1) and (2), and as shown in Fig. 4 , the shape of the $\Delta B_{s}-B_{0}$ curve and its initial gradient $\mathrm{d} B_{0} /\left.\mathrm{d} \Delta B_{s}\right|_{\Delta B_{s}=0}$ do not depend on $J_{c}$ but on the geometrical parameters. This was also true in the numerical calculation even when the depth of the layer in which the current flowed had a distribution in the $z$ direction inside the bulk, as shown by the numerical $\Delta B_{s}-B_{0}$ curve in Fig. 9. The only exception is the case that the current distribution at each different $T$ had a different shape for an equal $\Delta B_{s} / \Delta B_{s, \max }$. This is caused when the shape of the $J_{c}-B$ curve changes markedly with $T$. However, we know from the dc magnetization measurement that the shape of the $J_{c}-B$ curve did not change markedly with $T$ and also this effect did not appear in the initial gradient of the experimental $\Delta B_{s}-B_{0}$ curve in Fig. 9. Thus, by comparing the initial gradient, we can discuss the agreement without knowing the exact value of $J_{c}$.

From the comparison between the calculation and experiment, we clearly found that the results obtained by the EM method showed agreement with those of the experiment. The initial gradients of the curves in the experiment and the EM method are identical, whereas the initial gradient in the $\mathrm{CF}$ method is 1.7 times larger than that in the experiment. Moreover, the dependence curve of $B_{0}$ on $\Delta B_{s}$ in the calculation by the EM method is similar to that in the experiment. From the comparison, we can estimate the range of the average $J_{c}$ in the experiment. Because the average $J_{c}$ changes with the field, it is difficult and meaningless to estimate the exact value to fit the curve. The estimated average ranges of $J_{c}$ were $5-10 \mathrm{kA} / \mathrm{mm}^{2}$ at $T=20 \mathrm{~K}, 2-3 \mathrm{kA} / \mathrm{mm}^{2}$ at $T=40 \mathrm{~K}$, and approximately $1 \mathrm{kA} / \mathrm{mm}^{2}$ at $T=60 \mathrm{~K}$. These ranges are almost within the ranges from $J_{c}(B=0)$ to $J_{c}(B=2 \mathrm{~T})$ in Table I, and are thus thought to be reasonable. From these findings, we can conclude that the results of the EM method show agreement with those of the experiment. Additionally, we found that the macroscopic $J_{c}$ in the bulk HTSs was almost equal to that of the small sample. This means that the large degradation due to the difficulty of manufacturing large bulk HTSs or cracking did not occur.

The reason for the agreement is next discussed. The geometrical parameters $N, \lambda_{u}, g, D_{x}, D_{y}$, and $D_{z}$ and the bulk shape were equal in the experiment, the $\mathrm{CF}$ method, and the EM method. As stated in Sec. III A, the total amounts of current inside the bulk at the same $\Delta B_{s}$ are only slightly different for the two calculation methods. The only remaining factor is the geometrical shape of the current, i.e., the current distribution inside the bulk. Therefore, we conclude the following. The reason why the EM method closely reproduced the experiment is that it closely reproduced the current distribution in the experiment. The current distribution inside the bulk must be considered to precisely estimate the field inside the BHSAU.

We next discuss the results shown in Fig. 9. On the basis of the above discussion, the calculation was performed by the EM method with $J_{c}=5$ and $10 \mathrm{kA} / \mathrm{mm}^{2}$. The difference between the results obtained experimentally and by calculation for both $J_{c}$ is less than 5\% except near the peak at $z \sim-32 \mathrm{~mm}$. By considering the fact that the measurement has an error of $4 \%$, the difference of $5 \%$ is not large. The difference near the peak at $z \sim-32 \mathrm{~mm}$ is about $30 \%$. There are two possible reasons for this large difference. One is that the individual differences in $J_{c}$ among the bulk HTSs particularly affect peaks near the ends of the undulator. The other is that the current distribution cannot be reproduced well near the ends of the undulator even by the EM method. The former effect can be included in the calculation by including the individual differences in $J_{c}$ among the bulk HTSs. For the latter case, it is difficult to measure the actual current distribution inside the bulk HTSs. However, the difference can be evaluated by comparing a much larger amount of data, i.e., the $B_{y}$ and $B_{z}$ distributions in three-dimensional space near the ends of the undulator. If a difference appears, the assumption that 
the current flows only in the plane perpendicular to the $z$ axis should be changed in a future calculation.

Although the amplitude of the undulator field can be obtained by the empirical formulas derived in the next section, the numerical code remains useful for future research. As PMs have individual differences in magnetization, bulk HTSs also have differences in their critical current density, which cause the errors in the undulator field, such as so-called phase error and residual field integrals. Methods of compensating for these errors are practically important and the next topic of our research. It is not difficult to modify the numerical code to treat individual differences in the critical current density of bulk HTSs. Therefore, the numerical code can be used to study methods of compensating for these errors.

\section{Empirical formulas}

Next we modify the analytical formulas by using the results obtained by comparing the numerical and analytical calculations. The maximum $B_{0}$ in the numerical calculation is equal to that in the analytical calculation; therefore, the target range of the modification is the reasonable operation range discussed previously, and modification should be applied to the formula for $\Delta B_{s}$ but not to the formula for $B_{0}$ :

$$
\begin{gathered}
B_{0}^{\mathrm{Nu}}=B_{0}^{\mathrm{An}}, \\
\Delta B_{s}^{\mathrm{Nu}}=(1+\alpha) \Delta B_{s}^{\mathrm{An}} .
\end{gathered}
$$

As shown in Fig. 7, the factor $\alpha$ is at most 50\%. However, if a suitable $f$ is chosen for the target operation point, it can be less than $5 \%$. We fit the curves by the empirical formula,

$$
\alpha=a+b \frac{B_{0}^{\mathrm{An}}}{B_{0, \max }^{\mathrm{An}}}+c \exp \left(d \frac{B_{0}^{\mathrm{An}}}{B_{0, \max }^{\mathrm{An}}}\right)
$$

(for $a, b, c$, and $d$, see).

The fitting results of the parameters $a, b, c$, and $d$ are shown in Table IV. The error of the formula is less than $1 \%$ in the effective range shown in Table IV.

Additionally, we consider the effect of the periodic number. Because we used $N=12$ in the calculation for Fig. 7, the required $\Delta B_{s}$ should be modified as

$$
\Delta B_{s}^{\mathrm{Nu}}(N)=\beta(N) \times(1+\alpha) \Delta B_{s}^{\mathrm{An}} .
$$

Here, $\beta$ is a function of the periodic number, and the right term of the multiplication is the value when $N=12$. By fitting the dependence curve of $\Delta B_{s}$ on $N$ for $B_{0} / B_{0, \max }^{\mathrm{An}}=50 \%$, we have
TABLE IV. Parameters for Eq. (17).

\begin{tabular}{cccccc}
\hline \hline \multicolumn{5}{c}{$a+b x+c \exp (d x)$} & \\
$f$ & $a$ & $b$ & $c$ & $d$ & $\begin{array}{c}\text { Effective range } \\
B_{0} / B_{0, \max }[\%]\end{array}$ \\
\hline 0.55 & 0.296 & -0.294 & $\ldots$ & $\ldots$ & $20-90$ \\
0.60 & 0.176 & -0.188 & $7.93 \times 10^{-9}$ & 18.2 & $20-90$ \\
0.65 & 0.0995 & -0.107 & $1.50 \times 10^{-5}$ & 11.2 & $20-80$ \\
0.70 & 0.0551 & -0.0346 & $1.47 \times 10^{-4}$ & 9.89 & $20-70$ \\
\hline \hline
\end{tabular}

$$
\beta(N)=1.098-1.060 \frac{1}{N}-1.519 \frac{1}{N^{2}} .
$$

The error of this formula is less than $1 \%$ in the range of $N \geq 6$ and $20 \leq B_{0} / B_{0, \max }^{\mathrm{An}} \leq 90$.

Equation (18) reproduces the numerical $B_{0}$ and $\Delta B_{s}$ with an error of less than $2 \%$ for $D_{y}=2 \lambda_{u}, g / \lambda_{u}=0.4, N \geq 6$, and the effective $B_{0} / B_{0, \max }$ range shown in Table IV. Moreover, because it is clear from Table III that the shape of the $\Delta B_{s}-B_{0}$ curve has a difference of less than $2 \%$ among various values of $g / \lambda_{u}$, this modified formula can be applied to a wide range of conditions $\left(0.1 \leq g / \lambda_{u} \leq 10\right)$ with an error of less than $3 \%$.

\section{E. Usage of formulas and performance estimation}

Here we estimate the magnetic performance of the BHSAU as an example of using the formulas and the values obtained above. As examples of performance estimation, we calculated two patterns with $\lambda_{u}=18 \mathrm{~mm}$ and $g=3.6 \mathrm{~mm}$ by targeting a field twice as large as that generated in the IVU but with an equal period and gap. Next, we calculated two patterns with $\lambda_{u}=10 \mathrm{~mm}$ and $g=4 \mathrm{~mm}$ by targeting $K=2$ and two patterns with $\lambda_{u}=5 \mathrm{~mm}$ and $g=1 \mathrm{~mm}$ by targeting $K=1$ and 2 . Here, $K$ is called the deflection parameter and is defined by $K=e B_{0} \lambda_{u} /(2 \pi m c)$, where $e$ and $m$ are the electron charge and mass, respectively. To obtain bright undulator radiation, $K \sim 1-2$ is required.

In Table $\mathrm{V}$, we show the estimated $B_{0}$, and the values of $\Delta B_{s}$ and the operation temperature $T$ required to obtain each $B_{0}$. Here, $\Delta B_{s}$ is the value for $N=50$. Note that $\Gamma_{y}$ is not the actual depth of the layer in which the current flows and is simply the index in Table III. We set $J_{c}^{\text {bulk }}, \Gamma_{y}, f$, and

TABLE V. Performances of BHSAU.

\begin{tabular}{lcccccccc}
\hline \hline $\begin{array}{l}\lambda_{u} \\
{[\mathrm{~mm}]}\end{array}$ & $\begin{array}{c}g \\
{[\mathrm{~mm}]}\end{array}$ & $\begin{array}{c}J_{c}^{\text {bulk }} \\
{\left[\mathrm{kA} / \mathrm{mm}^{2}\right]}\end{array}$ & $\begin{array}{c}\Gamma_{y} \\
{[\%]}\end{array}$ & \multicolumn{1}{c}{$f$} & $\begin{array}{c}B_{0} \\
{[\mathrm{~T}]}\end{array}$ & $\begin{array}{c}\Delta B_{s} / 2 \\
{[\mathrm{~T}]}\end{array}$ & $\begin{array}{c}T \\
{[\mathrm{~K}]}\end{array}$ & $\mathrm{K}$ \\
\hline \multirow{2}{*}{18} & \multirow{2}{*}{3.6} & 2.4 & 30 & 0.6 & 2.6 & 4.8 & 40 & 4.4 \\
& & 7.1 & 5 & 0.7 & 2.6 & 2.3 & 20 & 4.4 \\
10 & 4 & 8.0 & 20 & 0.6 & 2.1 & 6.0 & 20 & 2.0 \\
& 4 & 12.0 & 10 & 0.65 & 2.1 & 4.4 & 10 & 2.0 \\
5 & \multirow{2}{*}{1} & 7.1 & 30 & 0.6 & 2.1 & 3.9 & 20 & 1.0 \\
& & 16.7 & 20 & 0.6 & 4.2 & 6.0 & 4 & 2.0 \\
\hline \hline
\end{tabular}


$T$ to satisfy the following conditions: (i) $\Delta B_{s} / 2$ does not exceed $6 \mathrm{~T}$, (ii) $J_{c}^{\text {bulk }}$ is in the range from $J_{c}(B=0)$ to $J_{c}\left(B=\Delta B_{s} / 2\right)$ at $T$, (iii) $f$ is chosen to obtain a small $\alpha$. Here, we assumed the field cooling method, i.e., the undulator is cooled to below the superconducting transition temperature under a solenoid field of $-\Delta B_{s} / 2$ and operated under a solenoid field of $\Delta B_{s} / 2$. Thus, we used $J_{c}^{\text {bulk }}$ in the range between $J_{c}(B=0)$ and $J_{c}\left(B=\Delta B_{s} / 2\right)$.

As shown in the table, an undulator field twice as large as that of the present IVU but with an equal period and gap can be obtained at temperatures of approximately $20-40 \mathrm{~K}$. Moreover, $K=1$ or 2 can be achieved with a short period such as 5 or $10 \mathrm{~mm}$. Because $B_{0}$ of over $4 \mathrm{~T}$ is required to achieve $K=2$ at $\lambda_{u}=5 \mathrm{~mm}$, it is impossible to achieve this using PM undulators even with $g=0$. Although $T=$ $4 \mathrm{~K}$ is required to achieve the condition of the bottom row in Table V with currently available bulk HTSs, if the critical current density of the bulk HTS is doubled as a result of future material developments, the BHSAU can be operated at approximately $20 \mathrm{~K}$. We believe that the magnetic field strength of the BHSAU is sufficiently high for future highfield short-period undulators.

\section{CONCLUSION}

The magnetic field inside a BHSAU was calculated by analytical and numerical methods to reveal its characteristics. The analytical calculation showed that a high undulator field could be achieved by the BHSAU at a high critical current density and that good undulator field tunability was maintained even in the saturation region. The numerical calculation by the EM method, which treats the current distribution inside the bulk HTSs, produced a considerably different current distribution from that obtained by the CF method, and closely reproduced the experimental results. Thus, we found that consideration of the current distribution is extremely important in calculating the undulator field and the required change in the solenoid field. To precisely estimate the fields without a large computation cost, by comparison between the analytical and numerical calculations, we obtained a semiempirical formula for the required solenoid field by modifying the analytical formula so that it treats the effect of the current distribution inside bulk HTSs. Finally, we estimated the magnetic performance of the BHSAU as an example of using the formulas and the values obtained in the discussion. The estimation showed that an undulator field twice as large as that of the present IVU but with an equal period and gap can be obtained at a temperature of approximately $20-40 \mathrm{~K}$, and that $K=1$ and 2 can be achieved with $\lambda_{u}=5$ and $10 \mathrm{~mm}$ at approximately $4-20 \mathrm{~K}$. We consider that the magnetic field strength of the BHSAU is sufficiently high for future high-field short-period undulators.

\section{ACKNOWLEDGMENTS}

This work was supported by MEXT KAKENHI Grant No. 21340057 and a Grant-in-Aid for JSPS Fellows, Grant No. $10 J 03477$.

[1] P. M. Stefan, T. Tanabe, S. Krinsky, G. Rakowsky, L. Solomon, and H. Kitamura, J. Synchrotron Radiat. 5, 417 (1998).

[2] T. Schmidt, G. Ingold, A. Imhof, B. D. Patterson, L. Patthey, C. Quitmann, C. Schulze-Briese, and R. Abela, Nucl. Instrum. Methods Phys. Res., Sect. A 467, 126 (2001).

[3] J. A. Clarke, in Proceedings of the European Particle Accelerator Conference, Vienna, Austria, 2000, p. 2319.

[4] O. Chubar, C. Benabderrahmane, M.P. Level, O. Marcouille, and M. Massal, in Proceedings of the 9th European Particle Accelerator Conference, Lucerne, 2004 (EPS-AG, Lucerne, 2004), p. 369.

[5] P. Emma et al., Nat. Photonics 4, 641 (2010).

[6] M. Hoener et al., Phys. Rev. Lett. 104, 253002 (2010).

[7] T. Ishikawa et al., Nat. Photonics 6, 540 (2012).

[8] J. Dai, H. Deng, and Z. Dai, Phys. Rev. Lett. 108, 034802 (2012).

[9] D. J. Scott, J. A. Clarke, D.E. Baynham, V. Bayliss, T. Bradshaw, G. Burton, A. Brummitt, S. Carr, A. Lintern, J. Rochford, O. Taylor, and Y. Ivanyushenkov, Phys. Rev. Lett. 107, 174803 (2011).

[10] H. Kitamura, J. Synchrotron Radiat. 7, 121 (2000).

[11] T. Hara, T. Tanaka, H. Kitamura, T. Bizen, X. Maréchal, T. Seike, T. Kohda, and Y. Matsuura, Phys. Rev. ST Accel. Beams 7, 050702 (2004).

[12] T. Hezel, B. Krevet, H. O. Moser, J. A. Rossmanith, R. Rossmanith, and T. Schneider, J. Synchrotron Radiat. 5, 448 (1998).

[13] M. Tomita and M. Murakami, Nature (London) 421, 517 (2003).

[14] T. Tanaka, T. Hara, H. Kitamura, R. Tsuru, T. Bizen, X. Marechal, and T. Seike, Phys. Rev. ST Accel. Beams 7, 090704 (2004).

[15] T. Tanaka, R. Tsuru, and H. Kitamura, J. Synchrotron Radiat. 12, 442 (2005).

[16] T. Tanaka, T. Hara, R. Tsuru, D. Iwaki, T. Bizen, X. Marechal, T. Seike, and H. Kitamura, Supercond. Sci. Technol. 19, S438 (2006).

[17] R. Kinjo, T. Kii, H. Zen, K. Higashimura, K. Masuda, K. Nagasaki, and H. Ohgaki, in Proceedings of the 30th International Free Electron Laser Conference, Geongju, Korea, 2008, p. 473.

[18] A. H. Ho, R. H. Pantell, J. Feinstein, and B. Tice, Nucl. Instrum. Methods Phys. Res., Sect. A 296, 631 (1990).

[19] R. Kinjo, M. Shibata, T. Kii, H. Zen, K. Masuda, K. Nagasaki, and H. Ohgaki, Appl. Phys. Express 6, 042701 (2013).

[20] K. Halbach, Nucl. Instrum. Methods Phys. Res. 187, 109 (1981).

[21] O. Chubar, P. Elleaume, and J. Chavanne, J. Synchrotron Radiat. 5, 481 (1998). 
[22] M. Morita, E. Teshima, and H. Hirano, Nippon Steel Corporation Technical Report No. 93, 2006.

[23] C. P. Bean, Rev. Mod. Phys. 36, 31 (1964).

[24] A. Badía, C. López, and J. L. Giordano, Phys. Rev. B 58, 9440 (1998).
[25] A. Sanchez and C. Navau, Phys. Rev. B 64, 214506 (2001).

[26] J. Kitagaki, K. Masuda, Z. W. Dong, T. Kii, T. Yamazaki, and K. Yoshikawa, Nucl. Instrum. Methods Phys. Res., Sect. A 475, 613 (2001). 